\title{
A simple, high-throughput, colourimetric, field applicable loop-mediated isothermal amplification (HtLAMP) assay for malaria elimination
}

\author{
Sumudu Britton ${ }^{1,2^{*}}$, Qin Cheng ${ }^{3}$, Colin J. Sutherland ${ }^{4}$ and James S. McCarthy ${ }^{1,2}$
}

\begin{abstract}
Background: To detect all malaria infections in elimination settings sensitive, high throughput and field deployable diagnostic tools are required. Loop-mediated isothermal amplification (LAMP) represents a possible field-applicable molecular diagnostic tool. However, current LAMP platforms are limited by their capacity for high throughput.

Methods: A high-throughput LAMP (HtLAMP) platform amplifying mitochondrial targets using a 96-well microtitre plate platform, processing 85 samples and 11 controls, using hydroxynaphtholblue as a colourimetric indicator was optimized for the detection of malaria parasites. Objective confirmation of visually detectable colour change results was made using a spectrophotometer. A dilution series of laboratory-cultured 3D7 Plasmodium falciparum parasites was used to determine the limit of detection of the HtLAMP assay, using P. falciparum (HtLAMP-Pf) and Plasmodium genus (HtLAMP-Pg) primers, on whole blood and filter paper, and using different DNA extraction protocols. The diagnostic accuracy of HtLAMP was validated using clinical samples from Papua New Guinea, Malaysia, Ghana and The Gambia and its field applicability was evaluated in Kota Marudu district hospital, Sabah, Malaysia.

Results: The HtLAMP assay proved to be a simple method generating a visually-detectable blue and purple colour change that could be objectively confirmed in a spectrophotometer at a wavelength of $600 \mathrm{~nm}$. When compared with PCR, overall HtLAMP-Pg had a sensitivity of $98 \%(n=260 / 266,95 \% \mathrm{Cl} 95-99)$ and specificity $83 \%(n=15 / 18$, $95 \% \mathrm{Cl} 59-96)$. HtLAMP-Pf had a sensitivity of $97 \%(n=124 / 128,95 \% \mathrm{Cl} 92-99)$ and specificity of $96 \%(n=151 / 157$, $95 \%$ Cl 92-99). A validation study in a regional hospital laboratory demonstrated ease of performance and interpretation of the HtLAMP assay. HtLAMP-Pf performed in this field setting had a sensitivity of $100 \%(n=17 / 17,95 \% \mathrm{Cl}$ $80-100)$ and specificity of $95 \%(n=123 / 128,95 \% \mathrm{Cl}$ 90-98) compared with multiplex PCR. HtLAMP-Pf also performed well on filter paper samples from asymptomatic Ghanaian children with a sensitivity of $88 \%$ ( $n=23 / 25,95 \%$ Cl 69-97).
\end{abstract}

Conclusion: This colourimetric HtLAMP assay holds much promise as a field applicable molecular diagnostic tool for the purpose of malaria elimination.

Keywords: Loop mediated isothermal amplification, LAMP, Elimination, High throughput, Diagnosis, Malaria

\section{Background}

Malaria elimination is an ambitious but enticing goal. Elimination is defined as "interrupting local

\footnotetext{
*Correspondence: sumudu.britton@qimrberghofer.edu.au

${ }^{2}$ QIMR Berghofer Medical Research Institute, Brisbane, Australia

Full list of author information is available at the end of the article
}

mosquito-borne malaria transmission in a defined geographical area, i.e. zero incidence of locally contracted cases, although imported cases will continue to occur" [1]. By definition, malaria elimination is fundamentally different from malaria control, as the focus shifts from detection of symptomatic patients to detection and clearance of all infections. This includes active case detection 
of asymptomatic persons with sub-patent parasitaemia [2], subjects who have been shown to be able to support ongoing malaria transmission [3].

For $P$. falciparum and $P$. vivax, passive and active detection of infection in the field have relied on two diagnostic tools: thick and thin blood film microscopy, and immunochromatographic detection of circulating parasite antigens using rapid diagnostic tests (RDT). While expert microscopy has been shown to have $100 \%$ sensitivity at thick film parasite densities of 50 parasites $/ \mu \mathrm{L}$, sensitivity decreases significantly under operational field conditions [4]. Furthermore, studies in both Africa and Asia show that microscopy alone will fail to identify a substantial proportion of asymptomatic infections caused by all Plasmodium species, compared to detection by PCR [3]. It has been suggested that the proportion of submicroscopic infections is greater in areas where malaria elimination programmes are in place [5]. Such infections remain transmissible, and thus pose a threat to malaria elimination [6]. The success of malaria elimination programs will, therefore, critically depend on successful identification and treatment of all infected individuals. This requires high performance diagnostic tools, as outlined by the malERA consultative group [7]. The target sensitivity has recently been updated by WHO to specify a detection limit of 2 parasites $/ \mu \mathrm{L}$ [8], a level substantially lower than expert microscopy.

Loop-mediated isothermal amplification (LAMP) is a nucleic acid detection technology that has the potential to significantly improve malaria field diagnostics, particularly in elimination settings. LAMP differs from PCR in several critical ways. Firstly, it is an isothermal process relying on the Bacillus stearothermophilus (Bst) polymerase enzyme. It does not require the cyclical temperature changes [9] necessary for PCR. This facilitates field adaptation of LAMP platforms. Secondly, a positive LAMP reaction results in the formation of a magnesium pyrophosphate precipitate which can be visually detected, by turbidimetry [9] or using metal ion indicators, such as calcein [10], hydroxynaphthol blue [11] and pico-green [12]. LAMP end products have also been visualized using melt curve analysis [13], a bioluminescent output in real time (BART) [14], a lateral flow dipstick [15] and a portable fluorescence detection unit (realAmp) [16].

Since its first description in 2001 [9] LAMP has been applied to the detection of a wide variety of bacterial [17, 18], viral [19], fungal [20] and parasitic [21] organisms, in particular to facilitate diagnosis of infection in resource limited settings. There has been a significant amount of research effort invested into adapting LAMP for diagnosis of malaria. LAMP has been used for the detection of $P$. falciparum using crudely extracted DNA from whole blood [22], or using a rapid boil and spin method [23].
Subsequently, LAMP has been used to identify all Plasmodium species [24], including P. knowlesi $[25,26]$, and LAMP primers have been optimized to improve the sensitivity with which $P$. falciparum $[23,27]$ and $P$. vivax $[28$, 29] can be detected. Commercially available Loopamp kits (Eiken Chemical co) have been validated for detection of P. falciparum [30], including low density infections [31] and for the indirect detection of $P$. vivax using a combination of pan-genus and $P$. falciparum-specific LAMP primers [32]. However, as Hsiang et al. noted, obtaining a high throughput LAMP assay would appear to be an important step in making the technology more widely applicable [33] for the purpose of malaria elimination. Although assay time for most LAMP platforms is rapid at $60-90 \mathrm{~min}$, sample throughput is restricted by the platform used. For example, the LoopAmp kits (Eiken Chemical Co) used in the turbidimeter (Eiken Chemical Co) has a capacity of only 16 samples, while the kits used with a heatblock and ultraviolet lamp has capacity of 46 samples [31]. The demonstration that colorimetric detection of LAMP products provides acceptable diagnostic accuracy [11] suggests that this could be adapted to produce a multi-well, low-cost format for the detection of malaria parasites by LAMP.

This paper describes development of a platform for malaria LAMP that increases the throughput of the assay, and simplifies DNA extraction and sample analysis, resulting in improved field applicability. Validation of the platform was carried out by investigating its diagnostic accuracy using PCR as a gold standard as it is highly sensitive and widely available in reference laboratories for detecting low-level parasitaemia.

\section{Methods}

\section{Plasmodium parasite and clinical samples}

A tenfold dilution series of laboratory-cultured 3D7 $P$. falciparum parasites was prepared in $50 \%$ haematocrit blood using initial samples with parasitaemia adjusted to 1 and $0.5 \%$, as determined by microscopic examination of thin blood films. The number of parasites per microlitre was calculated using an estimate of $5 \times 10^{6}$ red cells/ $\mu \mathrm{L}$ to create a dilution series of samples ranging from 5000 to 0.5 parasites $/ \mu \mathrm{L}$. Four $5 \mu \mathrm{L}$ blood spots at each dilution were dried for $48 \mathrm{~h}$ on blotting paper (Whatman $3 \mathrm{MM}$ ) before being stored at $4{ }^{\circ} \mathrm{C}$ with desiccant. Whole blood was stored at $-20{ }^{\circ} \mathrm{C}$.

For assay validation, clinical samples were retrospectively made available from the following clinical trials: (1) filter paper samples $(n=25)$ from asymptomatic children from Ghana [31, 34], (2) whole blood samples $(\mathrm{n}=97)$ from symptomatic children from The Gambia [35], (3) filter paper samples $(n=42)$ from symptomatic persons from a high prevalence malaria region 
in Papua New Guinea (PNG) and (4) filter paper samples $(\mathrm{n}=145)$ from a case-control study [36] undertaken in region of mixed malaria species infections in Sabah, Malaysia. The inclusion and exclusion criteria for each of these studies are outlined in their respective publications.

The study protocols for each study were approved by the Ghana Health Service Ethics Committee (proposal \#GHS-ERC-08/7/10) and the Ethics Committee of the London School of Hygiene and Tropical Medicine (proposal \#5775), Joint Gambia Government/Medical Research Council Ethics Committee (re. 2002/910), QIMR Berghofer Medical Research Institute Human Research Ethics Committee (P2119), the Malaysian Medical Research Ethics Committee and Menzies School of Health Research, respectively.

\section{DNA extraction from filter paper}

The chelex method for DNA extraction from filter paper [37] was further optimized to improve turnaround time. Briefly, $6 \mathrm{~mm}$ filter paper disc punches, corresponding to $5 \mu \mathrm{L}$ of blood, were incubated in $0.5 \%$ saponin in PBS either overnight (chelex standard protocol) or for $2 \mathrm{~h}$ (chelex rapid protocol), centrifuged for $2 \mathrm{~min}$ at $4000 \mathrm{rpm}$, supernatant discarded, washed in PBS, centrifuged for $2 \mathrm{~min}$ at $4000 \mathrm{rpm}$, heated at $98{ }^{\circ} \mathrm{C}$ in $150 \mu \mathrm{L}$ of $6 \%$ chelex (Sigma Aldrich, CAS 8047-15-2) for $30 \mathrm{~min}$ and centrifuged for $3 \mathrm{~min}$ at $4000 \mathrm{rpm}$. The resultant $100 \mu \mathrm{L}$ supernatant was stored at $-20^{\circ} \mathrm{C}$.

\section{DNA extraction from whole blood}

For whole blood, the chelex method [38] was further evaluated in two modified protocols. Briefly, $5 \mu \mathrm{L}$ of whole blood was mixed with either $200 \mu \mathrm{L}$ of nucleasefree water for $20 \mathrm{~min}$ at room temperature (WB-chelex protocol) or $200 \mu \mathrm{L}$ of $0.5 \%$ saponin, and incubated at $37{ }^{\circ} \mathrm{C}$ for $30 \mathrm{~min}$ (WB-chelex saponin protocol). Samples were then centrifuged for $3 \mathrm{~min}$ at 10,000 rpm, supernatant discarded, the pellet heated at $98{ }^{\circ} \mathrm{C}$ in $150 \mu \mathrm{L}$ of $6 \%$ chelex for $30 \mathrm{~min}$ and centrifuged for $3 \mathrm{~min}$ at $10,000 \mathrm{rpm}$. The resultant supernatant was stored at $-20^{\circ} \mathrm{C}$. For both extraction protocols, three volumes of blood, 5, 10 and $20 \mu \mathrm{L}$, were assessed.

\section{DNA extraction from clinical samples}

The Gambia whole blood samples were extracted using the WB-chelex protocol on $5 \mu \mathrm{L}$ of blood. The filter paper samples from Ghana were extracted using the FP-chelex standard protocol. The PNG and Sabah filter paper samples were extracted using the FP-chelex rapid protocol. The differences in methods used for clinical sample processing reflect the different sample sets used to validate each of the extraction protocols.

\section{High-throughput LAMP (HtLAMP) method}

High throughput (HtLAMP) was performed in a 96-well microtitre plate as described [11], with some modifications. The assays were performed in a standard $\mathrm{u}$-bottom microtitre plate (Sterihealth) covered with a clear disposable plate cover (Eppendorf). Each plate was able to process 85 samples allowing for 9 negative and 2 positive controls. Reactions were performed in $25 \mu \mathrm{L}$ total volume containing $1 \mathrm{X}$ buffer $(20 \mathrm{mM}$ Tris HCL pH 8.8, $\left.10 \mathrm{mM} \mathrm{KCl}, 8 \mathrm{mM} \mathrm{MgSO} 4,10 \mathrm{mM} \mathrm{NH}_{4} \mathrm{SO}_{4}\right), 1.25 \mathrm{mM}$ each dNTP, $3.55 \mu \mathrm{M}$ each Pf869FIP/BIP, $1.78 \mu \mathrm{M}$ each Pf869LF/LBn, $0.2 \mu \mathrm{M}$ each Pf869F3/B3, $1.18 \mu \mathrm{M}$ each PgID19FIP/BIP, $0.59 \mu \mathrm{M}$ each PgID19 LF/LB and $0.15 \mu \mathrm{M}$ each PgID19 F3/B3, $120 \mu \mathrm{M}$ Hydroxynaphtholblue (Fluka, CAS number 63451-35-4) and 8 units Bst polymerase (New England Biolabs, Ipswich, MA, USA). Primer sequences for Pf869 and PgID19 were as published by Polley et al. [23] except Pf869LBn GTTGAGA TGGAAACAGCCGG. Microtitre plates were incubated in a water bath at $65^{\circ} \mathrm{C}$ for 40 min before the colour change and precipitate in each well were recorded. Blue colour change with a visible precipitate was read as a positive result and purple colour change without a precipitate was read as a negative result (Fig. 1). Positive and negative samples were read by a single person who was blinded to the multiplex PCR results for the samples being analysed.

Performance of HtLAMP in a laboratory setting with limited resources involved storing reagents in a freezer at $-20{ }^{\circ} \mathrm{C}$ and preparation of the mastermix as required for performance of each HtLAMP assay. Efforts to prevent contamination were based on physical separation of DNA extraction from mastermix preparation areas and spectrophotometry, combined with stringent attention to

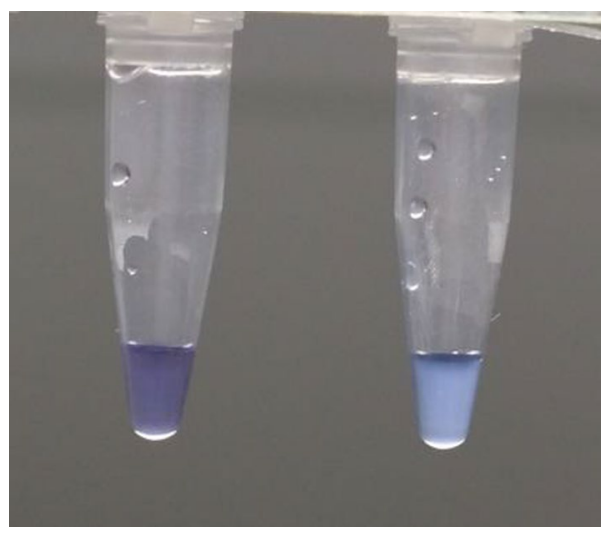

Fig. 1 HtLAMP colour change associated with hydroxynaphtholblue (HNB). Left clear, purple colour, without precipitate $=$ negative. Right Blue colour change with cloudy precipitate $=$ positive 
hand hygiene and glove and gown changes between the different assay areas.

\section{Limit of detection of HtLAMP}

The limit of detection (LOD) for the HtLAMP assay was determined by performing HtLAMP-Pg and HtLAMPPf on duplicate samples at each dilution. Filter paper extracted using the two DNA extraction protocols and whole blood 5, 10 and $20 \mu \mathrm{L}$ extracted using the two DNA extraction protocols were evaluated. The LOD was determined to be the lowest dilution (containing the least parasite material) at which both duplicates were positive by HtLAMP.

\section{Factors affecting Hydroxynaphthol blue (HNB)}

$\mathrm{HNB}$ is a metal ion indicator that is sensitive to changes in $\mathrm{pH}$ as well as concentrations of the divalent cations magnesium and calcium. Using $\mathrm{pH}$ calibration buffers at $\mathrm{pH}$ 4.0, 7.0 and 10.0 plus hydrochloric acid, 8 samples of different $\mathrm{pH}$ were produced and tested with HNB. Resulting colour changes were recorded visually. Magnesium sulphate (MW $246.68 \mathrm{~g} / \mathrm{mol}$ ) and calcium chloride (MW $110.99 \mathrm{~g} / \mathrm{mol}$ ) were used to make 5 samples of concentrations varying from 1 to $0.001 \mathrm{M}$. These were also tested with HNB and resulting colour changes were recorded visually.

\section{Spectrophotometer for determining threshold of positive result}

A plate spectrophotometer (PowerwaveXS2 software version 2.00.18) was used in order to determine a threshold value to objectively differentiate a positive from a negative HtLAMP result and demonstrate the reliability of visually detectable colour change in determining a positive and negative sample. Using a P. falciparum 3D7 DNA dilution series, HtLAMP mastermix reagents both with and without HNB were placed in the microtitre plate. Nuclease free water was used as the no template negative control. Following incubation in a water bath at $65^{\circ} \mathrm{C}$ for $40 \mathrm{~min}$, the microtitre plate was read visually to document cloudy and blue positive reactions and clear and purple negative reactions in the wells containing HNB. The presence and absence of cloudy white precipitate was recorded in the wells without HNB. The microtitre plate was then read in the spectrophotometer across the visible light spectrum from 300 to $700 \mathrm{~nm}$ to identify the wavelengths that best discriminated positive and negative samples both with and without HNB.

The optical density (OD) value with the best discrimination of $\mathrm{HNB}$ related positive and negative colour change as well as presence and absence of magnesium pyrophosphate precipitate was then used to read each well. The threshold value for a positive reaction was calculated using the mean plus two standard deviations of the no template control (NTC) wells. This threshold value was then subtracted from the OD reading for each well to obtain a positive or negative numerical value. The presence of a visible blue colour change with presence of cloudy precipitate was mandatory for a sample to be positive. A positive numerical OD value was used to confirm the visually determined positive result. A sample that was purple and clear was considered a negative result with a negative OD value confirming this result. Samples with only a positive OD result without corresponding colour change and precipitate were considered negative. Therefore, results were recorded based on their colour change with OD values being used to confirm the visually detected result. Only samples with the appropriate colour change and corresponding OD result were considered true positives or true negatives for the purpose of statistical analysis.

\section{Nested PCR}

For both whole blood and filter paper DNA extraction methods, the limit of detection was determined by comparison of HtLAMP to nested PCR as described by Snounou et al. [39]. PCR reactions were performed in $20 \mu \mathrm{L}$ total volume containing $1 \mathrm{X}$ buffer, $2 \mathrm{mM} \mathrm{MgCl} 2,200 \mu \mathrm{M}$ dNTPs, $200 \mu \mathrm{M}$ primer mix (rPLU5new/rPLU6 for nest 1 and rFAL1/rFAL2 for nest 2) and $0.5 \mathrm{U}$ Taq polymerase (Bioline). The PCR amplification products were visualized by gel electrophoresis.

\section{Multiplex PCR}

PCR was used as the reference standard for analysing the diagnostic accuracy of the HtLAMP platform given its sensitivity. Multiplex PCR (mPCR), which has an LOD of $0.02-0.05$ parasites $/ \mu \mathrm{L}$ as described by Padley et al. [40], and had already been performed on these samples as part of their respective studies, was used as the gold standard reference test for comparison of HtLAMP-Pf and HtLAMP-Pg in the analysis of the PNG and Sabah samples. Nested PCR [39] was performed on the Ghana and Gambia samples.

\section{Statistics}

Sensitivity and specificity of HtLAMP was calculated against $\mathrm{mPCR}$ and nested PCR. Sensitivity $=$ true positives/(true positives + false negatives) and specificity $=$ true negatives/(true negatives + false positives). The $95 \%$ confidence intervals were calculated using an online calculation tool [41].

\section{Feasibility of field application of htLAMP}

The HtLAMP assay field applicability was assessed by performing the assay in a regional hospital in Kota 
Marudu district in Sabah, Malaysia. DNA was extracted from filter paper samples of 145 symptomatic patients using the FP-chelex rapid protocol, assayed using HtLAMP-Pg and HtLAMP-Pf and resulted were interpreted visually and confirmed with a portable spectrophotometer. Following a 3-day training workshop for local malaria staff on performance and interpretation of HtLAMP assay results, a questionnaire was completed on challenges of performing the HtLAMP assay in a regional laboratory setting.

\section{Results}

\section{Limits of detection of HtLAMP}

The HtLAMP limit of detection was dependent on sample type, i.e. whole blood or filter paper, DNA extraction method and the LAMP primer used (Plasmodium genus primers PgID19 or P. falciparum primers Pf869 [23]) (Table 1). In the HtLAMP platform, overall, the Plasmodium genus LAMP primers (HtLAMP-Pg) performed with a better analytical sensitivity than the $P$. falciparum LAMP primers (HtLAMP-Pf). For whole blood 10 and 20 $\mu \mathrm{L}$, the chelex-saponin DNA extraction protocol resulted in better analytical sensitivity compared to using chelex alone. The explanation for the discrepancy in results for whole blood $5 \mu \mathrm{L}$ for the two extraction protocols are unclear but may include pipetting error of blood at small volumes and partial loss of red cell pellet during the DNA extraction process. The best analytical sensitivity for HtLAMP-Pg was achieved using $10 \mu \mathrm{L}$ of whole blood with the WB-chelex saponin protocol. This had a LOD of 2.5 parasites $/ \mu \mathrm{L}$ which was equal to the nested PCR used. The best LOD for HtLAMP-Pf was 5 parasites $/ \mu \mathrm{L}$ using $20 \mu \mathrm{L}$ of whole blood with the WB-chelex saponin protocol. This was equal to the LOD for HtLAMP-Pg at this volume, while nested PCR had an LOD of 0.5 parasite/ $\mu \mathrm{L}$.

For filter paper, the standard chelex protocol, with an overnight incubation step performed better than the rapid protocol with a $2 \mathrm{~h}$ incubation step, providing a tenfold lower (LOD) for HtLAMP-Pg, i.e. 2.5 parasites $/ \mu \mathrm{L}$ compared to 25 parasites $/ \mu \mathrm{L}$. For filter paper samples, the LOD for HtLAMP-Pg using the FP-standard chelex protocol for DNA extraction was 2.5 parasites $/ \mu \mathrm{L}$, while the sensitivity of HtLAMP-Pg using the FP-rapid chelex protocol was 25 parasites $/ \mu \mathrm{L}$. The HtLAMP-Pf assay was able to detect 5 parasites $/ \mu \mathrm{L}$ using the FP-standard chelex protocol and 50 parasites/ $\mu \mathrm{L}$ using the FP-rapid chelex protocol. Therefore, the FP rapid chelex protocol appears to trade-off analytical sensitivity for a faster turnaround time for the assay. Taken together, these results confirm that the starting sample material and method of DNA processing have important effects on the analytical sensitivity of the HtLAMP assay.

In the interest of improving turnaround time of the assay the FP- rapid chelex protocol was chosen despite a compromise in analytical sensitivity for the validation of the HtLAMP platform on filter paper samples from PNG and field testing in Sabah.

\section{Optimal wavelength for developing an objective threshold} for positive results

When interrogated across the visible spectrum of light, wavelengths between 600 and $650 \mathrm{~nm}$, appeared to best distinguish positive from negative samples, whether the cloudy magnesium pyrophosphate precipitate was present or not and whether there was a blue or purple colour change associated with HNB (Fig. 2). The wavelength of $600 \mathrm{~nm}$ was chosen to provide OD values for the HtLAMP assay for the following reasons; (a) this value produced OD values that discriminated well between cloudy and blue positive HtLAMP reactions and clear and purple negative samples in order to calculate an objective OD threshold for each plate of samples and (b) a $600 \mathrm{~nm}$ filter is a standard setting for many bench top spectrophotometers. Table 2 shows the correlation between the visually detected colour change and

Table 1 Summary of limits of detection for HtLAMP-Pg and HtLAMP-Pf for whole blood and filter paper with different DNA extraction protocols

\begin{tabular}{|c|c|c|c|c|}
\hline Sample $(n=4)$ & DNA extraction protocol & $\begin{array}{l}\text { Limit of detection } \\
\text { nested PCR (parasites/ } \mu \mathrm{L} \text { ) }\end{array}$ & $\begin{array}{l}\text { Limit of detection } \\
\text { HtLAMP-Pg (parasites/ } \mu \mathrm{L} \text { ) }\end{array}$ & $\begin{array}{l}\text { Limit of detection } \\
\text { HtLAMP-Pf (parasites/ } \mu \mathrm{L} \text { ) }\end{array}$ \\
\hline \multirow[t]{2}{*}{ Whole blood $5 \mu \mathrm{L}$} & WB-chelex & 5 & 5 & 50 \\
\hline & WB-chelex-saponin & 25 & 50 & 50 \\
\hline \multirow[t]{2}{*}{ Whole blood $10 \mu \mathrm{L}$} & WB-chelex & 2.5 & 25 & 50 \\
\hline & WB-chelex-saponin & 2.5 & 2.5 & 50 \\
\hline \multirow[t]{2}{*}{ Whole blood $20 \mu \mathrm{L}$} & WB-chelex & 25 & 50 & 50 \\
\hline & WB-chelex-saponin & 0.5 & 5 & 5 \\
\hline \multirow[t]{2}{*}{ Filter paper $5 \mu \mathrm{L}$} & $\begin{array}{l}\text { Chelex standard } \\
\text { protocol (overnight) }\end{array}$ & 2.5 & 2.5 & 5 \\
\hline & Chelex rapid protocol (2 h) & 5 & 25 & 50 \\
\hline
\end{tabular}




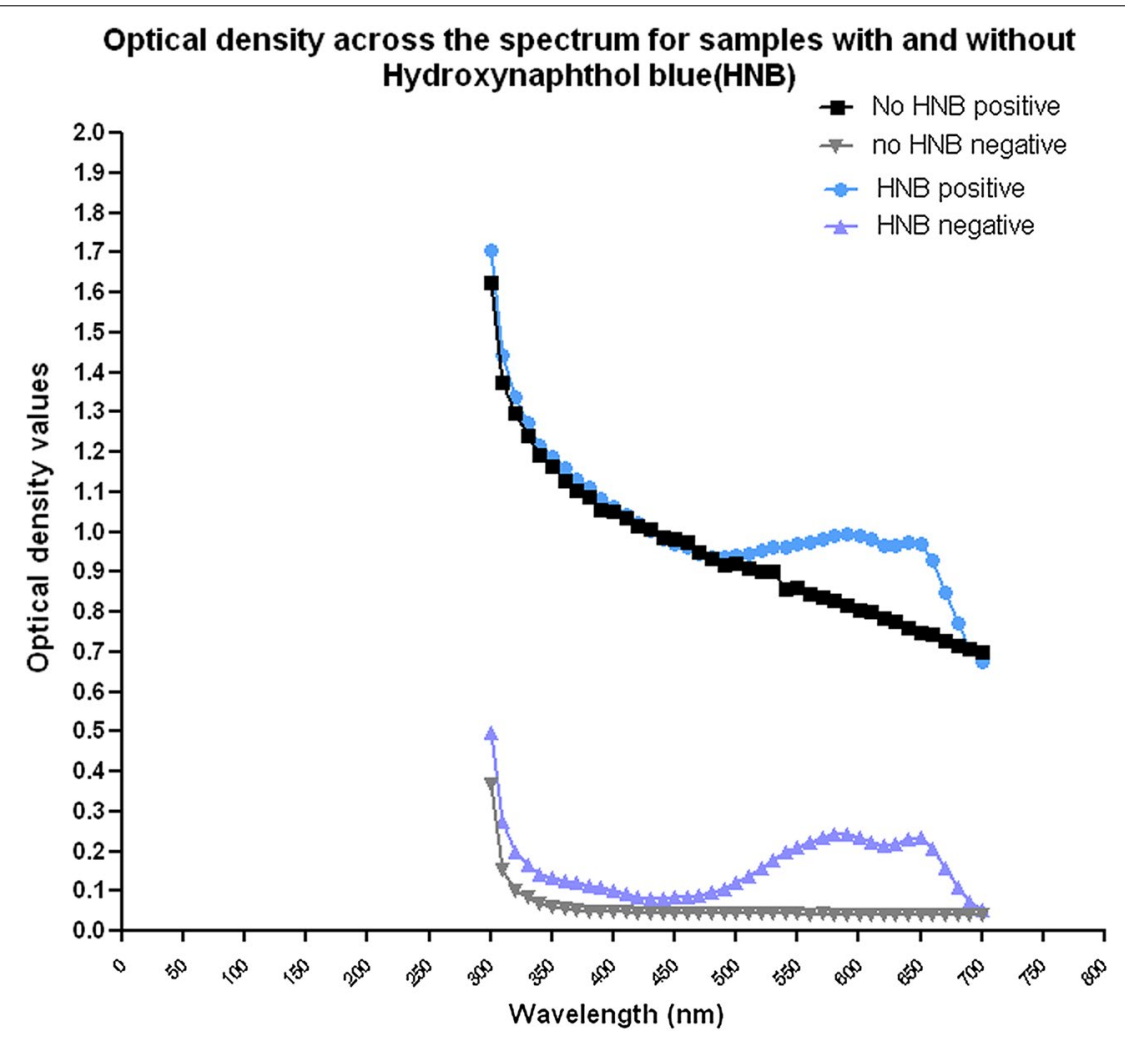

Fig. 2 Optical density values across the visible spectrum of light for HtLAMP samples with and without HNB. Between the wavelengths of 550-650 nm higher optical densitometry readings were found for samples that were positive by HtLAMP (no HNB positive and HNB positive). In addition, at these wavelengths, the optical densitometry readings were also able to distinguish samples that were positive due to the presence of the magnesium pyrophosphate precipitate (no HNB positive) and samples that were by positive by HtLAMP due to the blue colour change associated (HNB positive)

corresponding OD-derived threshold values for the interpretation of HtLAMP-Pf results for a subset of samples from the field validation study in Sabah.

\section{Effect of $\mathrm{pH}$, magnesium sulphate and calcium chloride on HNB colour change}

The colour change associated with HNB was significantly affected by changes in $\mathrm{pH}$, magnesium and calcium. At pH 3, HNB had a pink appearance compared with dark blue at $\mathrm{pH} 7$ and sky blue at $\mathrm{pH} 10$. Colour variation was also seen with magnesium, ranging from pink at a concentration of $0.001 \mathrm{M}$ concentration to dark purple at a concentration of $1.0 \mathrm{M}$, and calcium, from dark pink at $0.01 \mathrm{M}$ concentration to bright blue at $0.5 \mathrm{M}$ concentrations. This suggests that maintaining the correct concentration of calcium, magnesium and $\mathrm{pH}$ of the mastermix, as well as awareness of other metal ions present during extractions processes, is important in allowing HNB to undergo its colour change during a LAMP reaction.

\section{Sensitivity and specificity of HtLAMP-Pg and HtLAMP-Pf} in clinical samples from symptomatic patients for the detection of $P$. falciparum

Filter paper blood spots from symptomatic patients from Papua New Guinea and Sabah, Malaysia and whole blood samples from The Gambia were evaluated using HtLAMP-Pg and HtLAMP-Pf to determine the diagnostic accuracy of the assay for the detection of $P$. falciparum. The features of each of these sample sets and composite sensitivity and specificity of HtLAMP$\mathrm{Pg} / \mathrm{Pf}$ for the detection of P. falciparum are outlined in Table 3.

Overall, HtLAMP-Pg had a sensitivity of $98 \%$ ( $n=260 / 266,95 \%$ CI 95-99) and specificity of $83 \%$ ( $\mathrm{n}=15 / 18,95 \%$ CI 59-96) for the detection of Plasmodium species. HtLAMP-Pf had a sensitivity of $97 \%$ ( $\mathrm{n}=124 / 128,95 \%$ CI 92-99) and specificity of $96 \%$ (n $=151 / 157,95 \%$ CI 92-99) for the detection of $P$. falciparum. 
Table 2 HtLAMP-Pf optical densitometry (OD) results for 77 filter paper samples demonstrating the correlation between the visually detected colour change results for positive samples and their correlation with the positive results based on an ODderived threshold value

\begin{tabular}{|c|c|c|c|c|c|c|c|c|c|c|c|c|c|}
\hline & 1 & 2 & 3 & 4 & 5 & 6 & 7 & 8 & 9 & 10 & 11 & 12 & \\
\hline \multirow[t]{3}{*}{ A } & 29 & 154 & 19 & 17 & 22 & 27 & 34 & 23 & 180 & 32 & 42 & 176 & 0.225 \\
\hline & 0.374 & 0.229 & 0.256 & 0.257 & 0.219 & 0.217 & 0.252 & 0.244 & 0.344 & 0.243 & 0.296 & 0.333 & 0.235 \\
\hline & 0.112 & -0.033 & -0.006 & -0.005 & -0.043 & -0.045 & -0.01 & -0.018 & 0.082 & -0.019 & 0.034 & 0.071 & 0.218 \\
\hline \multirow[t]{3}{*}{$B$} & 46 & 30 & 35 & 66 & 36 & 47 & 41 & 52 & 48 & 186 & 53 & 54 & 0.224 \\
\hline & 0.248 & 0.327 & 0.234 & 0.342 & 0.238 & 0.241 & 0.239 & 0.23 & 0.217 & 0.216 & 0.231 & 0.366 & 0.263 \\
\hline & -0.014 & 0.065 & -0.028 & 0.08 & -0.024 & -0.021 & -0.023 & -0.032 & -0.045 & -0.046 & -0.031 & 0.104 & 0.215 \\
\hline \multirow[t]{3}{*}{$C$} & 65 & 51 & 72 & & 73 & 62 & 84 & 67 & 70 & 90 & 74 & 92 & 0.208 \\
\hline & 0.232 & 0.246 & 0.232 & 0.225 & 0.338 & 0.333 & 0.256 & 0.224 & 0.246 & 0.366 & 0.228 & 0.31 & 0.243 \\
\hline & -0.03 & -0.016 & -0.03 & -0.037 & 0.076 & 0.071 & -0.006 & -0.038 & -0.016 & 0.104 & -0.034 & 0.048 & 0.225 \\
\hline \multirow[t]{3}{*}{ D } & 77 & 96 & 81 & 85 & 194 & 89 & & 100 & 101 & 95 & 68 & 106 & 0.239 \\
\hline & 0.231 & 0.217 & 0.248 & 0.217 & 0.226 & 0.243 & 0.235 & 0.257 & 0.357 & 0.22 & 0.317 & 0.236 & 0.242 \\
\hline & -0.031 & -0.045 & -0.014 & -0.045 & -0.036 & -0.019 & -0.027 & -0.005 & 0.095 & -0.042 & 0.055 & -0.026 & 0.262 \\
\hline \multirow[t]{3}{*}{$E$} & 159 & & 122 & 131 & 124 & 127 & 94 & 136 & 129 & 105 & 187 & 142 & \\
\hline & 0.248 & 0.218 & 0.221 & 0.225 & 0.225 & 0.212 & 0.312 & 0.208 & 0.213 & 0.304 & 0.227 & 0.211 & \\
\hline & -0.014 & -0.044 & -0.041 & -0.037 & -0.037 & -0.05 & 0.05 & -0.054 & -0.049 & 0.042 & -0.035 & -0.051 & \\
\hline \multirow[t]{3}{*}{$F$} & 137 & 147 & 149 & 189 & & 141 & 112 & 146 & 157 & $112(1)$ & 160 & 198 & \\
\hline & 0.252 & 0.237 & 0.22 & 0.209 & 0.224 & 0.213 & 0.207 & 0.228 & 0.225 & 0.227 & 0.301 & 0.274 & \\
\hline & -0.01 & -0.025 & -0.042 & -0.053 & -0.038 & -0.049 & -0.055 & -0.034 & -0.037 & -0.035 & 0.039 & 0.012 & \\
\hline \multirow[t]{3}{*}{ G } & & 115 & & 116 & & 177 & 119 & 178 & & 181 & 123 & 151 & \\
\hline & 0.263 & 0.232 & 0.215 & 0.211 & 0.21 & 0.202 & 0.199 & 0.211 & 0.208 & 0.191 & 0.263 & 0.216 & \\
\hline & 0.001 & -0.03 & -0.047 & -0.051 & -0.052 & -0.06 & -0.063 & -0.051 & -0.054 & -0.071 & 0.001 & -0.046 & \\
\hline \multirow[t]{3}{*}{$\mathrm{H}$} & pos & pos & NTC & NTC & NTC & NTC & & & & & & & \\
\hline & 0.407 & 0.385 & 0.243 & 0.225 & 0.223 & 0.239 & 0.242 & 0.27 & 0.261 & 0.207 & 0.19 & 0.175 & \\
\hline & 0.145 & 0.123 & -0.019 & -0.037 & -0.039 & -0.023 & -0.02 & 0.008 & -0.001 & -0.055 & -0.072 & -0.087 & \\
\hline
\end{tabular}

Samples in blue had a blue/cloudy colour change as well as a corresponding positive OD threshold value. Samples in yellow were positive only by OD threshold. Shaded grey wells were negative controls within the plate. OD values for all negative control samples in each plate are used to calculate the OD threshold value (mean plus two standard deviations) which in this example is 0.262

Sensitivity and specificity of the combination of HtLAMP-Pg and HtLAMP-Pf for the detection of non-falciparum Plasmodium mono-infection in symptomatic patients

The diagnostic accuracy of the combination of HtLAMP-Pg and HtLAMP-Pf for the detection of non-falciparum Plasmodium species mono-infections was also assessed. In this context, the sensitivity of HtLAMP-Pg was $100 \%$ ( $\mathrm{n}=232 / 232,95 \%$ CI 98-100), with a specificity of $83 \%(n=15 / 18,95 \%$ CI 56-96).

Subgroup analysis demonstrated that in the Papua New Guinea samples, HtLAMP-Pg had a sensitivity of $100 \%$ ( $\mathrm{n}=9 / 9$, P. vivax positive) and specificity of $81 \%(n=13 / 16)$. There were 3 false positive HtLAMPPg results. HtLAMP-Pf was negative in all the monoinfection samples. For the 128 non-falciparum species infections from Sabah, HtLAMP-Pg had a sensitivity of $100 \%(\mathrm{n}=128 / 128)$. HtLAMP-Pf was negative for 122 of these samples. For the 6 false positive HtLAMPPf results (five $P$. vivax, one $P$. knowlesi) there was no evidence of $P$. falciparum co-infection by PCR. In the Gambia samples, of the 95 HtLAMP-Pg positive samples, only 1 was negative by HtLAMP-Pf, suggesting a non-falciparum species mono-infection which was confirmed by PCR to be $P$. malariae with a parasitaemia of 7.5 parasites $/ \mu \mathrm{L}$.

\section{Sensitivity of HtLAMP in persons with asymptomatic parasitaemia}

Features of the 25 samples chosen from a set of samples from asymptomatic school children in Kumasi, Ghana are outlined (see Additional file 1). Of the 25 samples $100 \%$ were positive for P. falciparum by nested PCR and $44 \%$ were co-infected with $P$. malariae. Interestingly, HtLAMP-Pg was positive for two samples in which only $P$. falciparum gametocytes were seen on microscopy of which one was also positive by HtLAMP-Pf. Compared with nested PCR the sensitivity of HtLAMP-Pg was $96 \%$ ( $\mathrm{n}=24 / 25,95 \%$ CI 80-100) and sensitivity of HtLAMPPf was $88 \%$ ( $\mathrm{n}=22 / 25,95 \%$ CI 69-97) (Table 4). HtLAMP-Pg and HtLAMP-Pf were able to detect the samples with the lowest parasitaemia of 40 parasites $/ \mu \mathrm{L}$ $(\mathrm{n}=3)$.

\section{Feasibility of field application of HtLAMP}

HtLAMP was performed in a regional hospital laboratory in Kota Marudu district, Sabah, Malaysian Borneo. This laboratory was able to perform basic haematology and biochemistry testing but did not perform microbiological culture or molecular assays. The HtLAMP assay was performed in a plastic bucket adapted to function as a water bath using a heating element and thermometer. A portable spectrophotometer (ChroMate) with a $600 \mathrm{~nm}$ 


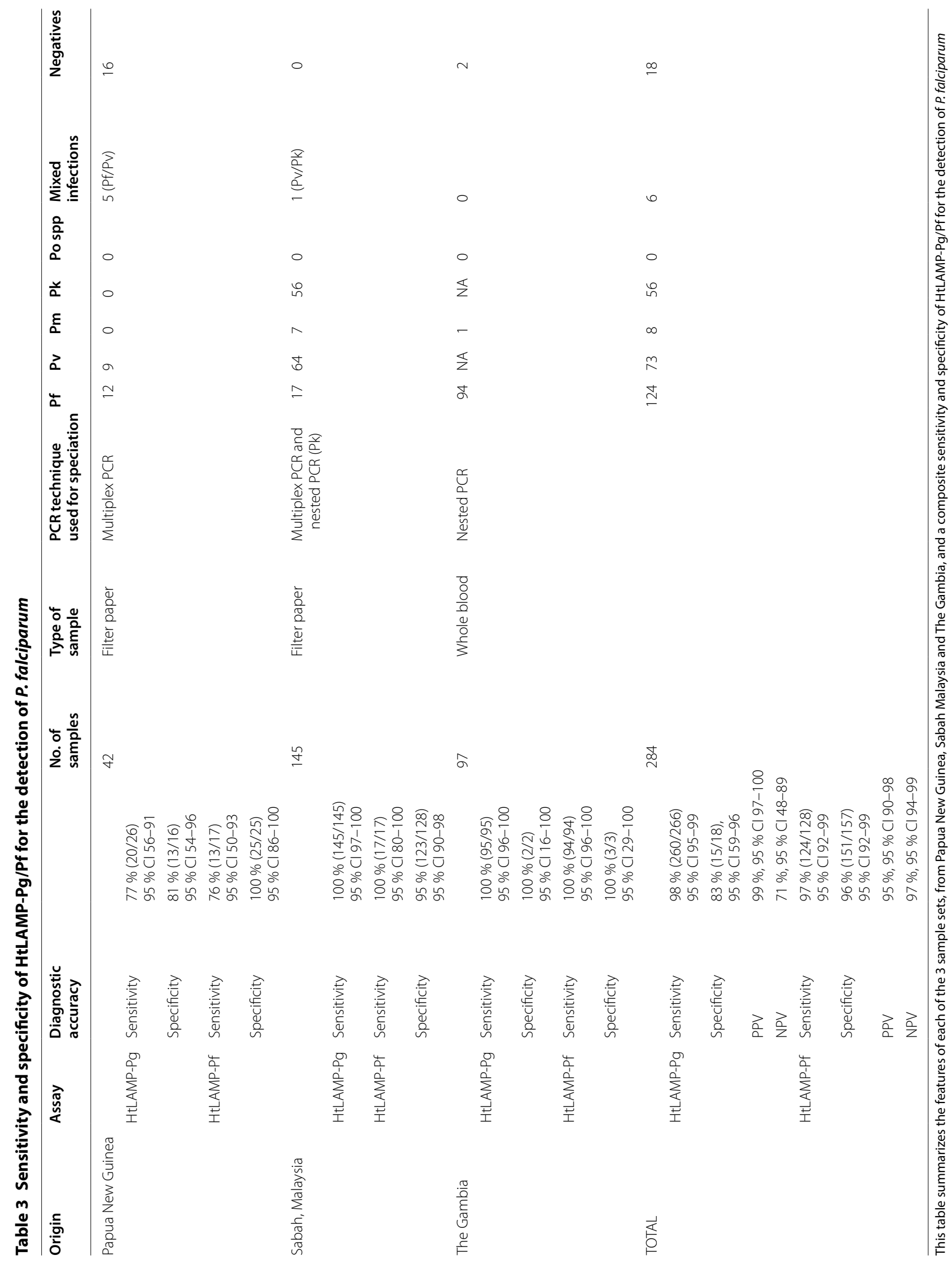


Table 4 Sensitivity and specificity of HtLAMP compared with nested PCR in Ghanaian children with asymptomatic parasitaemia

\begin{tabular}{lll}
\hline & Sensitivity & PPV \\
\hline HtLAMP-Pg (compared & $96 \%(24 / 25)$ & $100 \%$ \\
with nested PCR) & $95 \%$ Cl 80-100 & $95 \%$ Cl 85-100 \\
HtLAMP-Pf (compared & $88 \%(22 / 25)$ & $100 \%$ \\
with nested PCR) & $95 \%$ Cl 69-97 & $95 \%$ Cl 85-100 \\
\hline
\end{tabular}

filter provided the optical densitometry values. Samples and HtLAMP reagents were stored at $-20^{\circ} \mathrm{C}$ in a freezer. Three separate benches within a large room were dedicated to DNA extraction, HtLAMP assay setup and spectrophotometry in order to prevent cross-contamination.

The HtLAMP-Pg assay, performed in this resourcelimited laboratory setting, had a sensitivity of $100 \%$ ( $\mathrm{n}=145 / 145,95 \%$ CI 97-100). The HtLAMP-Pf assay had a sensitivity of $100 \%(\mathrm{n}=17 / 17,95 \%$ CI $80-100)$ and a specificity of $95 \%(n=123 / 128,95 \%$ CI 90-98).

Two local malaria laboratory staff was trained over a 3 day period to perform DNA extraction from filter paper and whole blood, setup the assay and interpret the results of the HtLAMP assay. Issues that were identified by local staff as potential limitations to deployment of HtLAMP in a field laboratory were difficulty of accurate sample placement into the 96-well plate and the availability and cost of reagents. However, it was felt that these limitations were balanced against the rapid turnaround time for the detection of parasites in microscopy negative samples thereby facilitating earlier patient treatment and household screening, potential time and cost saving in avoiding sending samples for PCR testing at reference laboratories and ease of interpretation of visual colour change results on the HtLAMP assay. The main challenges to setting up HtLAMP in this non-reference, low resource setting laboratory were ensuring good workflow to prevent contamination, and ensuring the reliability of ancillary equipment such as the centrifuge and freezers.

\section{Discussion}

To achieve its ambitious goals, the malaria elimination agenda will likely require diagnostic capacity to actively identify sub-patent infection in order to interrupt transmission. PCR, the most sensitive molecular diagnostic tool currently available, is limited by its technical requirements, particularly in resource limited field settings. A robust, low-cost and high-throughput assay for the rapid detection of malaria parasite DNA in finger-prick blood samples would provide this capacity. LAMP has been adapted into several different platforms [16, 31, 42-44] in order to improve its field applicability and throughput. While the definition of the throughput required for the application of LAMP for the purpose of malaria elimination remains to be established, the number of samples able to be processed simultaneously on these platforms range from $5[42]$ to $46[31,44]$ per assay.

The HtLAMP assay for the diagnosis of malaria that is described here has a simple platform-a standard 96-well microtitre plate, covered with a clear plate seal and incubated in a water bath at $65{ }^{\circ} \mathrm{C}$, that is able to process a minimum of 85 samples (allowing for positive and negative controls). The use of a colorimetric indicator enhances visual detection of the assay endpoint. To improve objectivity of interpretation of results, the assay uses a spectrophotometer at $600 \mathrm{~nm}$ to produce a threshold value which correlates with the visible colour change. In addition, DNA extraction protocols for whole blood and filter paper based on chelex and saponin have been modified to improve turnaround time while maintaining the cost effectiveness of the assay.

However, there appears to be significant variation in the LOD of HtLAMP depending on sample type, DNA extraction method and primers selected, particularly so for filter paper samples where there was a ten-fold loss of analytical sensitivity using a DNA extraction method that had a faster turnaround time. Nevertheless, HtLAMP-Pg using $10 \mu \mathrm{L}$ of whole blood with a chelex saponin protocol and HtLAMP-Pg on a $5 \mu \mathrm{L}$ filter paper blood spot using the chelex overnight protocol achieved a LOD of 2.5 parasites $/ \mu \mathrm{L}$. This suggests that the HtLAMP platform has the potential to reach the WHO target LOD. However, further modifications, such as using commercial kits, optimisation of low cost DNA extraction methods or using a larger volume of blood are likely to be required to achieve the balance between improved analytical sensitivity and turnaround time. The availability of more sensitive LAMP primers to detect Plasmodium genus and species- specific primers may also enhance the LOD of the HtLAMP assay. Therefore, malaria elimination programs choosing diagnostic tools may be required to make important operational and logistics decisions with regards to both the type of assay used and the sample types, for example whole blood or filter paper, that can achieve the best analytical sensitivity for that assay in order to meet the current WHO target of 2 parasites $/ \mu \mathrm{L}$.

The HtLAMP assay has been validated in a resource limited laboratory setting using filter paper samples and the chelex rapid DNA extraction protocol, despite its aforementioned limitations, in order to evaluate its turnaround time in this clinical setting. Compared with PCR, which requires samples to be sent to a reference laboratory, HtLAMP-Pg had a sensitivity of $100 \%$ and HtLAMP-Pf had a sensitivity of $100 \%$ and a specificity of $95 \%$ and results were available within $24 \mathrm{~h}$. 
In this study, the HtLAMP assay was validated for the detection of Plasmodium genus and P. falciparum on clinical samples from microscopy positive symptomatic and asymptomatic patients from regions with different Plasmodium species prevalence. Overall, HtLAMP-Pg had a sensitivity of $98 \%$ and specificity of $83 \%$ and HtLAMP-Pf had a sensitivity of $97 \%$ and specificity of $96 \%$ for the detection of P. falciparum. The use of the combination of Plasmodium genus and P. falciparum LAMP primers showed a HtLAMP-Pg sensitivity of $100 \%$ and specificity $83 \%$ compared to PCR for the detection of non-falciparum Plasmodium monoinfections. These results are comparable to other LAMP studies in symptomatic patients (Table 5). However, further studies are required to determine the sensitivity and specificity of HtLAMP in detecting sub-microscopic infections in asymptomatic patients.

The 96 well HtLAMP platform is able to test 85 samples per plate, allowing for positive and negative controls. The cost per test in this platform is USD\$ 0.85 excluding primers and DNA extraction. Final cost will depend on the starting material (filter paper or whole blood), the use of either crude or commercial DNA extraction protocols and the primer sets selected. Nevertheless, the cost per sample to perform HtLAMP-Pg on whole blood extracted using chelex-saponin protocol is USD\$1. The turnaround time for completion of an assay, including DNA extraction, is $4-6 \mathrm{~h}$.

However, HtLAMP is not a quantitative assay, a limitation it shares with other LAMP based assays. Other potential limitations include the use of HNB, which is sensitive to changes in $\mathrm{pH}$ and metal ion concentrations. Awareness of, for example, the type of DNA extraction method used on samples, which may contain reagents that affect pH and metal ion concentrations, would help maintain the reliability of interpretation of the colour change aspect of the assay. Nevertheless, this is balanced against its low cost, stability at room temperature and easy visual interpretation of results. In its current format, HtLAMP relies on the storage of reagents at $-20^{\circ} \mathrm{C}$. While an important issue in terms of field deployment, this could potentially be overcome by lyophilization of reagents.

\section{Conclusion}

The HtLAMP platform offers several important improvements over currently available LAMP platforms. Firstly, HtLAMP is able to process up to 85 samples simultaneously which makes it an ideal potential platform for surveillance or household surveys. Secondly, it uses standard consumables, and can be performed using DNA extracted by chelex and saponin based protocols which makes it very cost effective. Thirdly, one of the significant advantages to the use of HNB is that it can be added to the start of the assay such that HtLAMP remains a closed system, thereby reducing the risk of cross-contamination. Fourthly, while the platform of HtLAMP has been used here with Plasmodium genus and P. falciparum primers, it could be adapted to detect any other blood-borne pathogen of interest, thereby broadening the potential applications of the platform beyond the diagnosis of malaria. Finally, HtLAMP has been performed successfully in a regional laboratory setting by local staff trained over a very short period of time demonstrating its ease of performance and interpretation of results.

This provides confidence that the HtLAMP could be usefully deployed as a molecular diagnostic tool to support malaria elimination in resource-limited settings.

Table 5 Comparison of sensitivity and specificity of HtLAMP-Pg and HtLAMP-Pf for the detection of P. falciparum and non-falciparum species with other LAMP studies in symptomatic patients

\begin{tabular}{|c|c|c|c|c|c|}
\hline LAMP study & Sample source & Species & LAMP primer & Sensitivity (\%) & Specificity (\%) \\
\hline \multirow[t]{2}{*}{ HtLAMP } & \multirow[t]{2}{*}{ Symptomatic patients } & \multirow[t]{2}{*}{ P. falciparum } & $\mathrm{Pg}$ & 98 & 83 \\
\hline & & & Pf & 97 & 96 \\
\hline \multirow[t]{2}{*}{ Polley et al. [23] } & \multirow[t]{2}{*}{ Symptomatic patients } & \multirow[t]{2}{*}{ P. falciparum } & $\mathrm{Pg}$ & 93.9 & 100 \\
\hline & & & Pf & 93.3 & 100 \\
\hline \multirow[t]{2}{*}{ Polley et al. [27] } & \multirow[t]{2}{*}{ Symptomatic returned travellers } & \multirow[t]{2}{*}{ P. falciparum } & $\mathrm{Pg}$ & 97 & 99.2 \\
\hline & & & Pf & 98.4 & 98.1 \\
\hline Hopkins et al. [30] & Passive case detection & P. falciparum & Pf & 89-93.9 & $76.3-85$ \\
\hline HtLAMP & Symptomatic patients & Non-falciparum & $\mathrm{Pg}$ & 100 & 83 \\
\hline Vallejo et al. [32] & Symptomatic patients & Non-falciparum & $\mathrm{Pg}$ & 91.4 & 91.8 \\
\hline
\end{tabular}




\section{Additional file}

Additional file 1: Features of asymptomatic Ghanaian children samples $(n=25)$. Table outlines features of the 25 samples chosen from a set of samples from asymptomatic school children in Kumasi, Ghana.

\section{Authors' contributions}

SB carried out all the laboratory work, SB, CJS, JMC and QC conceived of and helped develop the platform, provided clinical samples for its validation and helped draft the manuscript. The opinions expressed herein are those of the authors and do not necessarily reflect those of the Australian Defence Force Joint Health Command. All authors read and approved the final manuscript.

\section{Author details}

${ }^{1}$ University of Queensland, Brisbane, Australia. ${ }^{2}$ QIMR Berghofer Medical Research Institute, Brisbane, Australia. ${ }^{3}$ Australian Army Malaria Institute, Brisbane, Australia. ${ }^{4}$ London School of Hygiene and Tropical Medicine, London, UK.

\section{Acknowledgements}

Thank you to all institutions for the collection and provision of samples used for assay validation. Thank you to Nicholas Anstey, Matthew Grigg and Timothy William for facilitating field evaluation of the assay. CJS is supported by Public Health England, the Medical Research Council, UK, and the Malaria Eradication Scientific Alliance. SB is funded by a National Health and Medical Research Council (NHMRC) Australia scholarship and a Royal Brisbane Hospital Foundation scholarship.

\section{Compliance with ethical guidelines}

\section{Competing interests}

The authors declare that they have no competing interests.

Received: 7 July 2015 Accepted: 11 August 2015

Published online: 28 August 2015

\section{References}

1. WHO. Global malaria control and elimination: report of a technical review. Geneva: World Health Organization; 2008.

2. WHO. Disease surveillance for malaria elimination: an operational manual. Geneva: World Health Organization; 2012.

3. Okell LC, Bousema T, Griffin JT, Ouedraogo AL, Ghani AC, Drakeley CJ. Factors determining the occurrence of submicroscopic malaria infections and their relevance for control. Nat Commun. 2012:3:1237.

4. Coleman RE, Maneechai N, Rachaphaew N, Kumpitak C, Miller RS, Soyseng $\mathrm{V}$, et al. Comparison of field and expert laboratory microscopy for active surveillance for asymptomatic Plasmodium falciparum and Plasmodium vivax in western Thailand. Am J Trop Med Hyg. 2002;67:141-4.

5. Okell LC, Ghani AC, Lyons E, Drakeley CJ. Submicroscopic infection in Plasmodium falciparum-endemic populations: a systematic review and meta-analysis. J Infect Dis. 2009;200:1509-17.

6. Bousema T, Drakeley C. Epidemiology and infectivity of Plasmodium falciparum and Plasmodium vivax gametocytes in relation to malaria control and elimination. Clin Microbiol Rev. 2011;24:377-410.

7. MalERA Consultative Group on Diagnoses and Diagnostics. A research agenda for malaria eradication: diagnoses and diagnostics. PLoS Med 2011; 8:e1000396.

8. WHO. WHO evidence review group on malaria diagnosis in low transmission settings, 16-18 December 2013. Geneva: World Health Organization; 2014

9. Mori Y, Nagamine K, Tomita N, Notomi T. Detection of loop-mediated isothermal amplification reaction by turbidity derived from magnesium pyrophosphate formation. Biochem Biophys Res Commun. 2001;289:150-4.

10. Tomita N, Mori Y, Kanda H, Notomi T. Loop-mediated isothermal amplification (LAMP) of gene sequences and simple visual detection of products. Nat Protoc. 2008;3:877-82.
11. Goto M, Honda E, Ogura A, Nomoto A, Hanaki K. Colorimetric detection of loop-mediated isothermal amplification reaction by using hydroxy naphthol blue. Biotechniques. 2009;46:167-72.

12. Wastling SL, Picozzi K, Kakembo ASL, Welburn SC. LAMP for Human African trypanosomiasis: a comparative study of detection formats. PLoS Negl Trop Dis. 2010;4:e865.

13. Yamamura M, Makimura K, Ota Y. Evaluation of a new rapid molecular diagnostic system for Plasmodium falciparum combined with DNA filter paper, loop-mediated isothermal amplification, and melting curve analysis. Jpn J Infect Dis. 2009;62:20-5.

14. Kiddle G, Hardinge P, Buttigieg N, Gandelman O, Pereira C, McElgunn CJ, et al. GMO detection using a bioluminescent real time reporter (BART) of loop mediated isothermal amplification (LAMP) suitable for field use. BMC Biotechnol. 2012;12:15.

15. Njiru ZK, Mikosza AS, Armstrong T, Enyaru JC, Ndung'u JM, Thompson AR. Loop-mediated isothermal amplification (LAMP) method for rapid detection of Trypanosoma brucei rhodesiense. PLoS Negl Trop Dis. 2008;2:e147.

16. Lucchi NW, Demas A, Narayanan J, Sumari D, Kabanywanyi A, Kachur SP, et al. Real-time fluorescence loop mediated isothermal amplification for the diagnosis of malaria. PLoS One. 2010;5:e13733.

17. Ou X, Li Q, Xia H, Pang Y, Wang S, Zhao B, et al. Diagnostic accuracy of the PURE-LAMP test for pulmonary tuberculosis at the county-level laboratory in China. PLoS One. 2014;9:e94544.

18. Dittrich S, Castonguay-Vanier J, Moore CE, Thongyoo N, Newton PN, Paris DH. Loop-mediated isothermal amplification for Rickettsia typhi (the causal agent of murine typhus): problems with diagnosis at the limit of detection. J Clin Microbiol. 2014;52:832-8.

19. Luo L, Nie K, Yang MJ, Wang M, Li J, Zhang C, et al. Visual detection of high-risk human papillomavirus genotypes $16,18,45,52$, and 58 by loopmediated isothermal amplification with hydroxynaphthol blue dye. J Clin Microbiol. 2011;49:3545-50

20. Scheel CM, Zhou Y, Theodoro RC, Abrams B, Balajee SA, Litvintseva AP. Development of a loop-mediated isothermal amplification method for detection of Histoplasma capsulatum DNA in clinical samples. J Clin Microbiol. 2014;52:483-8.

21. Arimatsu Y, Kaewkes S, Laha T, Sripa B. Specific diagnosis of Opisthorchis viverrini using loop-mediated isothermal amplification (LAMP) targeting parasite microsatellites. Acta Trop. 2015;141:368-71.

22. Poon LL, Wong BW, Ma EH, Chan KH, Chow LM, Abeyewickreme W, et al. Sensitive and inexpensive molecular test for falciparum malaria: detecting Plasmodium falciparum DNA directly from heat-treated blood by loop-mediated isothermal amplification. Clin Chem. 2006;52:303-6.

23. Polley SD, Mori Y, Watson J, Perkins MD, Gonzalez IJ, Notomi T, et al. Mitochondrial DNA targets increase sensitivity of malaria detection using loopmediated isothermal amplification. J Clin Microbiol. 2010;48:2866-71.

24. Han E, Watanabe R, Sattabongkot J, Khuntirat B, Sirichaisinthop J, Iriko $H$. Detection of four Plasmodium species by genus and species-specific loop mediated isothermal amplication for clinical diagnosis. J Clin Microbiol. 2007:45:2521-8.

25. Lau YL, Fong MY, Mahmud R, Chang PY, Palaeya V, Cheong FW, et al. Specific, sensitive and rapid detection of human Plasmodium knowlesi infection by loop-mediated isothermal amplification (LAMP) in blood samples. Malar J. 2011;10:197.

26. Iseki H, Kawai S, Takahashi N, Hirai M, Tanabe K, Yokoyama N, et al. Evaluation of a loop-mediated isothermal amplification method as a tool for diagnosis of infection by the zoonotic simian malaria parasite Plasmodium knowlesi. J Clin Microbiol. 2010;48:2509-14.

27. Polley SD, Gonzalez IJ, Mohamed D, Daly R, Bowers K, Watson J, et al. Clinical evaluation of a loop-mediated amplification kit for diagnosis of imported malaria. J Infect Dis. 2013;208:637-44.

28. Patel JC, Oberstaller J, Xayavong M, Narayanan J, Debarry JD, Srinivasamoorthy $\mathrm{G}$, et al. Real-time loop-mediated isothermal amplification (ReaLamp) for the species-specific identification of Plasmodium vivax. PLoS One. 2013;8:e54986.

29. Lu F, Gao Q, Zhou H, Cao J, Wang W, Lim CS, et al. Molecular test for vivax malaria with loop-mediated isothermal amplification method in central China. Parasitol Res. 2011;110:2439-44.

30. Hopkins H, Gonzalez IJ, Polley SD, Angutoko P, Ategeka J, Asiimwe C, et al. Highly sensitive detection of malaria parasitemia in a malaria-endemic setting: performance of a new loop-mediated isothermal amplification kit in a remote clinic in Uganda. J Infect Dis. 2013;208:645-52. 
31. Cook J, Aydin-Schmidt B, Gonzalez IJ, Bell D, Edlund E, Nassor MH, et al. Loop-mediated isothermal amplification (LAMP) for point-of-care detection of asymptomatic low-density malaria parasite carriers in Zanzibar. Malar J. 2015:14:43.

32. Vallejo AF, Martinez NL, Gonzalez IJ, Arevalo-Herrera M, Herrera S. Evaluation of the loop mediated isothermal DNA amplification (LAMP) kit for malaria diagnosis in P. vivax endemic settings of Colombia. PLoS Negl Trop Dis. 2015;9:e3453.

33. Hsiang MS, Greenhouse B, Rosenthal PJ. Point of care testing for malaria using LAMP, loop mediated isothermal amplification. J Infect Dis. 2014;210:1167-9.

34. Dinko B, Oguike M, Larbi J, Bousema T, Sutherland C. Persistent detection of Plasmodium falciparum, P. malariae, P. ovale curtisi and P. ovale wallikeri after ACT treatment of asymptomatic Ghanaian school-children. Int J Parasitol Drugs Drug Resist. 2013;3:45-50.

35. Sutherland CJ, Ord R, Dunyo S, Jawara M, Drakeley CJ, Alexander N, et al. Reduction of malaria transmission to Anopheles mosquitoes with a sixdose regimen of co-artemether. PLoS Med. 2005;2:e92.

36. Grigg MJ, William T, Drakeley CJ, Jelip J, von Seidlein L, Barber BE, et al. Factors that are associated with the risk of acquiring Plasmodium knowlesi malaria in Sabah, Malaysia: a case-control study protocol. BMJ Open. 2014;4:e006004.

37. Plowe CV, Djimde A, Bouare M, Doumbo O, Wellems TE. Pyrimethamine and proguanil resistance-conferring mutations in Plasmodium falciparum dihydrofolate reductase: polymerase chain reaction methods for surveillance in Africa. Am J Trop Med Hyg. 1995;52:565-8.
38. Walsh PS, Metzger DA, Higuchi R. Chelex 100 as a medium for simple extraction of DNA for PCR-based typing from forensic material. Biotechniques. 1991;10:506-13.

39. Snounou G, Viriyakosol S, Zhu XP, Jarra W, Pinheiro L, do Rosario VE, et al. High sensitivity of detection of human malaria parasites by the use of nested polymerase chain reaction. Mol Biochem Parasitol. 1993;61:315-20

40. Padley D, Moody AH, Chiodini PL, Saldanha J. Use of a rapid, single-round, multiplex PCR to detect malarial parasites and identify the species present. Ann Trop Med Parasitol. 2003;97:131-7.

41. MedCalc. http://www.medcalc.org/calc/diagnostic_test.php.

42. Sema M, Alemu A, Bayih A, Getie S, Getnet G, Guelig D, et al. Evaluation of non-instrumented nucleic acid amplification by loop-mediated isothermal amplification (NINA-LAMP) for the diagnosis of malaria in Northwest Ethiopia. Malar J. 2015;14:44.

43. Yongkiettrakul S, Jaroenram W, Arunrut N, Chareanchim W, Pannengpetch S, Suebsing R, et al. Application of loop-mediated isothermal amplification assay combined with lateral flow dipstick for detection of Plasmodium falciparum and Plasmodium vivax. Parasitol Int. 2014;63:777-84

44. Morris U, Khamis M, Aydin-Schmidt B, Abass AK, Msellem MI, Nassor MH, et al. Field deployment of loop-mediated isothermal amplification for centralized mass-screening of asymptomatic malaria in Zanzibar: a preelimination setting. Malar J. 2015;14:205.

\section{Submit your next manuscript to BioMed Central and take full advantage of:}

- Convenient online submission

- Thorough peer review

- No space constraints or color figure charges

- Immediate publication on acceptance

- Inclusion in PubMed, CAS, Scopus and Google Scholar

- Research which is freely available for redistribution

Submit your manuscript at

www.biomedcentral.com/submit

C Biomed Central 